\title{
Design offshore spherical tank support using shape optimization
}

\author{
Musaddiq $\mathrm{Al} \mathrm{Ali}{ }^{\mathrm{a}}$ * \\ ${ }^{a}$ Hiroshima University, 1-3-2 Kagamiyama, Higashi-Hiroshima City, 739-8511, Japan \\ *Corresponding Author: mosadeq007@yahoo.com
}

\begin{abstract}
The offshore spherical tank is used to store liquids of various types and hazardous factors. Such storage usually is in a dynamic way such that the liquid is relatively recirculated, and transferee. offshore spherical tank support needs time for designing and establishing. The advancement of rapid prototyping is a promising technology to be used in the near future, and it can give great results associated with non-parametric optimization. Weight minimization is the focus of structural optimization for several decades. The importance of weight minimization pushed structural optimization to take non-parametric optimization methodology. In this paper, shape optimization based on Level set method is used to achieve weight minimization for offshore spherical tank support. The level set method is a well-known method in image processing. It is been adapted to be as update methodology of the design domain in order to achieve the optimal design. Method of feasible directions was used for three objective functions, compliance, single stress, and pnorm function. Compliance function shows very good results comparing to the stress-based function. Feasible designs were produced from Level set method integration with the Optistruct solver.
\end{abstract}

Keywords: Level set method, Method of feasible direction, Shape Optimization.

\section{Introduction}

Structure design ${ }^{(1)}$ can be defined as the material arrangement which serves to resist mechanical loading. Generally, structural design is an optimization process in which, material selection, achieving high stiffness and minimizing maximum stress within the structure are the main goals. Add to that the economic factor which represents weight reduction. Structural optimization the methodology of determining best configuration of the problem physical reality. The best configuration is the best solution of well-chosen mathematical representation based on the principle of mechanics ${ }^{(2)}$. The formulation of the mechanical problem is an orthodox variational calculus ${ }^{(3)}$. Due to the advancement in computational methods, numerical discretization the mathematical problem is used effectively. Finite element method (FEM) is one of the widely used discretization method ${ }^{(4)}$ and used effectively with the non-parametric optimization. Spherical tanks are used for storing and transporting gases ${ }^{(5)}$. Spherical tanks are widely used for liquefied natural gas transport $(6,7)$. Support structure for spherical tanks is securing the position of the tank in both longitudinal and lateral directions. In case of dynamic loading, support structure should also additively suspend the movement. Structural optimization is mainly divided into the following three categories: Size (as an example of parametric optimization), shape and topology optimization. This paper is introducing topology optimization as a method of designing spherical tanks supports. In the static case, Topology optimization can give non-anticipated novel designs that focus on certain functionality effectively. For example, increasing fatigue life and smart suspension structure in case of vibration and at the same time, achieving lightweight structures. Shape optimization has been developed rapidly in last decades, and still a considerable attractive topic to be addressed due to free computer design. It based on the based auto design in order to find the optimal shape of the designed part based on updating the status of sub-domain within the design domain (support structure in the present case), such that the subdomain will take the optimal spatial configuration to construct the final optimal domain.

\section{Level set method}

Shape optimization is the part of structural optimization which deals with extremum structural boundaries. The shape is the term describing the outline of the structure, Mathematically the limit of the function by the first order 
gradient. In shape optimization, besides the objective function, shape representative is being chosen to address boundaries growth.
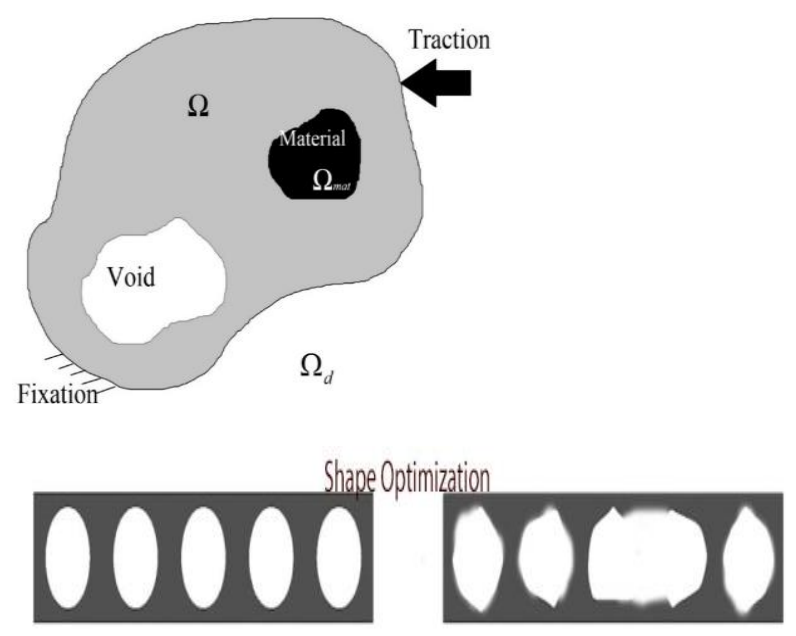

Fig. 1. Shape optimization process.

Level set method ${ }^{(8,9)}$, is one of the methodologies used to perform shape optimization. There are other shape optimization methodologies such as phase filed and Mesh morphing ${ }^{(10)}$. Phase field, and shape morphing face several challenges, leaving the level set method as the desired method due to its properties, and development. Level set optimization is one of shape optimization methodology which gains more interest recently. The level set method used as finite element adaptation method, which needs no re-mesh. For example; surface detachment ${ }^{(11-13)}$, and crack propagation analysis ${ }^{(14,15)}$ using extreme finite element method (XFEM). The level set is implicitly representing the domain boundary as the level set function $\varphi(\rho){ }^{(16)}$.

$\left\{\begin{array}{c}\varphi(\rho)>0: \rho \in \Omega / \partial \Omega \\ \varphi(\rho)=0: \rho \in \partial \Omega \\ \varphi(\rho)<0: \rho \in D / \partial \Omega\end{array}\right.$

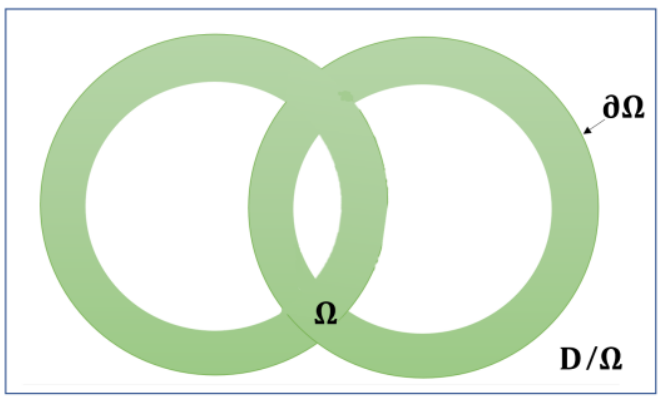

Fig. 2. Level set scheme.
The domain changing $\partial \Omega$ is done by normal velocity vector to the boundary $\frac{d \rho}{d t}$. The boundary motion is changing according to the Hamilton Jacobi equation.

$$
\frac{\partial \varphi(x)}{\partial t}+\nabla \varphi(x) \frac{d \rho}{d t}=0
$$

Explicitly, for the first instant, might lead to drawback which is introducing not assured regions if it been enclosed in sharp angle boundary. Add to that, boundary discretization might not supply sufficient segments that growth ca relay on ${ }^{(17)}$. Mathematical implementation has been introduced to improve level set method which overcomes the previously mentioned drawbacks ${ }^{(18)}$.

\section{Objective functions}

\subsection{Compliance optimization function}

Finite Element discretization of linear elastic continuum mechanics is taking the form of displacement approach as

$$
K(\rho) \mathbf{u}=\mathbf{F}
$$

Here, $\mathrm{K}$ is the structural stiffness matrix depending on density function. $\mathbf{u}$ is the nodal displacement vector, and $\mathbf{F}$ is nodal force vector. Stiffness is the measurement of structure to withstand certain load. It can be introducing in term of finite element analysis as its opposite; i.e. compliance. So, minimizing compliance will mean maximizing stiffness. Conditional objective function based on compliance is

$$
\begin{aligned}
& \lim _{\text {volf. } \rightarrow 0} \rho\left\{\Gamma\left(\Omega \cap \Omega_{\text {mat }}=0\right)\right\} \\
& \min ., \mathbf{F}^{T} \mathbf{u} \mid E \\
& \text { s.t. } \quad \int_{\Omega_{d}} \rho d \rho \leq V_{d}, 0<\rho_{\min }<\rho_{e}<1 \quad \forall \rho \in \Omega_{d}
\end{aligned}
$$

$V_{d}$ is a fraction of volume reduction, $\rho_{e}$ is elemental density, $\Omega$ is the whole domain including design and non-design domain $\Omega_{\text {mat }}$. The power $(p)$ that satisfies the condition of 2D SIMP set to be within ${ }^{(19)}$

$$
p \in \begin{cases}\left.\int \frac{4}{1+v}, \frac{2}{1-v}\right] & \text { in } 2 D \\ \left.115 \frac{1-v}{7-5 v}, \frac{31-v}{21-2 v}\right] & \text { in } 3 D\end{cases}
$$




\subsection{Stress based optimization function}

Stiffness maximization will not necessitate stress minimization. Compliance function is being used successfully for the past three decades.

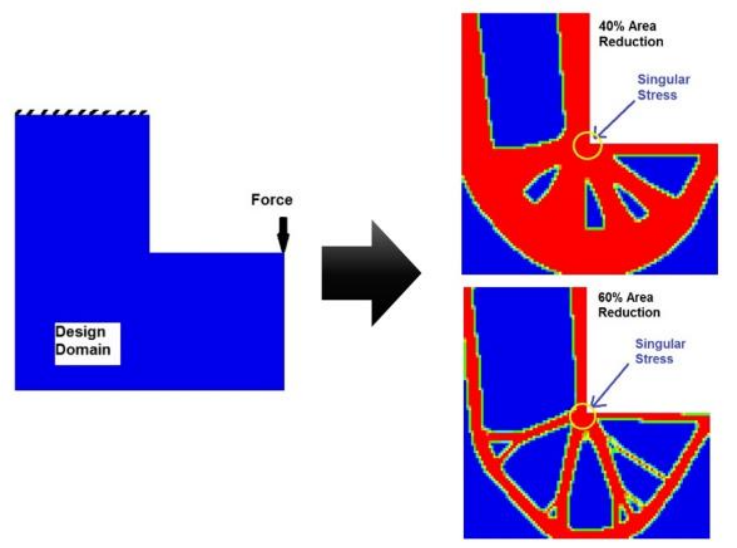

Fig. 3. Compliance objective function-based shape optimization

Stress can be addressed as an effective objective function to minimize the singularity topography (stress concentration parts). In this work, two stresses based objective function (SBOF) are addressed, first is single stress function, and the second one is the aggregative approach (p-norm function). Single stress is considerably easier to program and handled. However, it can be computationally costly. This approach identifies the maximum stress parts easily, yet singular stress parts can theoretically ${ }^{(20,21)}$, leads to a non-convergence solution so, the singularity is problem face topology optimization ${ }^{(20)}$. Elastic failure criteria are used as the averaging method which gives single stress to be measured in order to achieve a safe design. One of these yielding envelopes is the maximum shear strain energy per unit volume criteria which usually refer to with Maxwell von Mises stress $\left(\sigma_{v m s}\right)$ as in equation 47.

$\sigma_{\text {vms }}=\frac{1}{2} \sqrt{\left(\sigma_{x}-\sigma_{y}\right)^{2}+\left(\sigma_{y}-\sigma_{z}\right)^{2}+\left(\sigma_{z}-\sigma_{x}\right)^{2}+6\left(\tau_{x y}^{2}+\tau_{y z}^{2}+\tau_{z x}^{2}\right)}$

The maximum allowable von Mises stress could be identifying for certain material. In order to establish stress criterion as a valid objective function to be extremum, the relationship of scaled stress should be formed to satisfying the following; simplicity to decrease unnecessary commotions, physical coherence, and address material discretization directly. qp-approach is satisfying the above; which take the form

$$
\rho^{p-q}\left(\sigma_{a v g}\right) \mid \sigma_{v m s}
$$

p-norm stress function ${ }^{(22)}$ is used to overcome some of the limitations of single stress approach. This approach is based on choosing the Lebesgue space (equation 8) as continuous objective function ${ }^{(23)}$

$$
\ell^{P}(\Omega)=\left\{\sigma_{v m s} \in M(\Omega): \int_{\Omega}\left|\sigma_{v m s}(t)\right|^{P} d t\right\}
$$

With norm defined by

$$
\left\|\sigma_{v m s}\right\|_{P}=\left\{\begin{array}{c}
\sqrt[P]{\ell^{P}(\Omega)}, 1 \leq P<\infty \\
\sup \left\{\left|\sigma_{v m s}(\rho)\right| \rho \in[0,1]\right\}
\end{array}\right.
$$

Optimization will consider the first part of norm Equation 9. Theoretically, efficient optimization could be achieved for a higher value of $\mathrm{P}$ as the need for computational power. In other words, maximum stress region can be recognized by p-norm function with increasing the value of the power $\mathrm{P}$. This will lead to magnifying maximum stress of the system and then it be addressed intensively in the optimization process. The objective function that used is taking the form

$$
\left\{\begin{array}{c}
\text { find } \rho \rightarrow \rho^{p} \mid p=3 \\
\left\|\sigma_{v m s}\right\|_{P}=\sqrt[P]{\sum_{i=1}^{m}\left|\frac{\sigma_{v m s}}{\sigma_{\text {yeild }}}\right|^{P}} \\
\text { Extermum. },\left\|\sigma_{v m s}\right\|_{P} \mid E \\
\text { s.t. } \int_{\Omega_{d}} \rho d \rho \leq V_{d}, 0<\rho_{\min }<\rho_{e}<1 \quad \forall \rho \in \Omega_{d}
\end{array}\right.
$$

$\left\|\sigma_{v m s}\right\|_{P}$ is wildly known as P-norm function, or sometimes KK function refining to Park K. $(21,24)$ and Kikuchi associated with the appropriate constraint. Due to discretization nature of TO, mesh quality and type play vital role in pre-and post-processing of design. As mentioned previously, stress-based topology optimization affected by FEM accumulative analysis history. High order elements may increase the odds "theoretically "of better design, and higher resolution designs as well as increasing element geometric density. However, computational and time cost may be a serious problem along with convergence. Considering stress varying problem, the design of non-zero mean stress is done by calculating the safety factor to limit 
allowable working stress for the chosen design. Decreasing stress concentration areas is the strategy of increasing the allowable working stress.

\section{Design methodologies of spherical tanks support}

Spherical tank support is designed according to the tank specifications and the area to be established in. Most of the designer tenders are based on the existing structures (which is extensively studied for static and dynamic stabilities, and manufacturability).

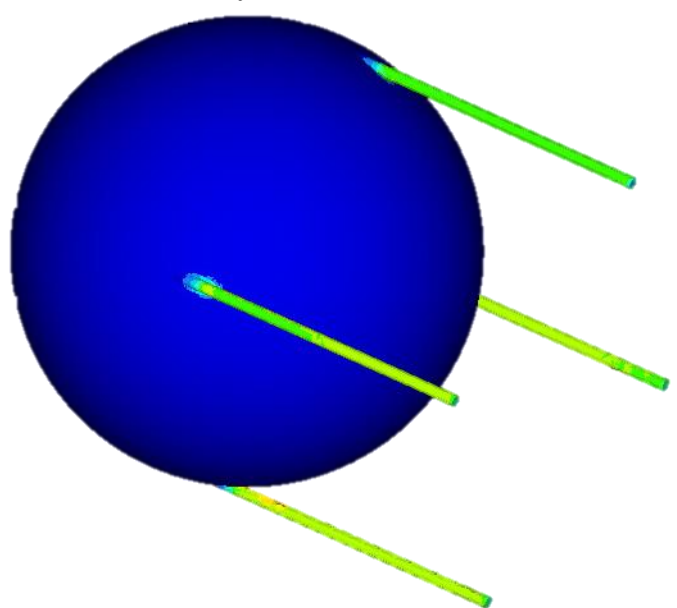

Fig. 4. Simple support design example.

Figure 4 shows high stress for the support tank interface. Topology optimization can gives a complex design to satisfy the complex objectives and boundary conditions for example; Conformal Lattice structure approach (CLS) is been introduce into the OptiStruc solver and been investigated by researchers ${ }^{(25-27)}$. CLS approached needs to use a complex linkage structures which add more complexity to establishing the structure in civil engineering as shown in Figure 5.

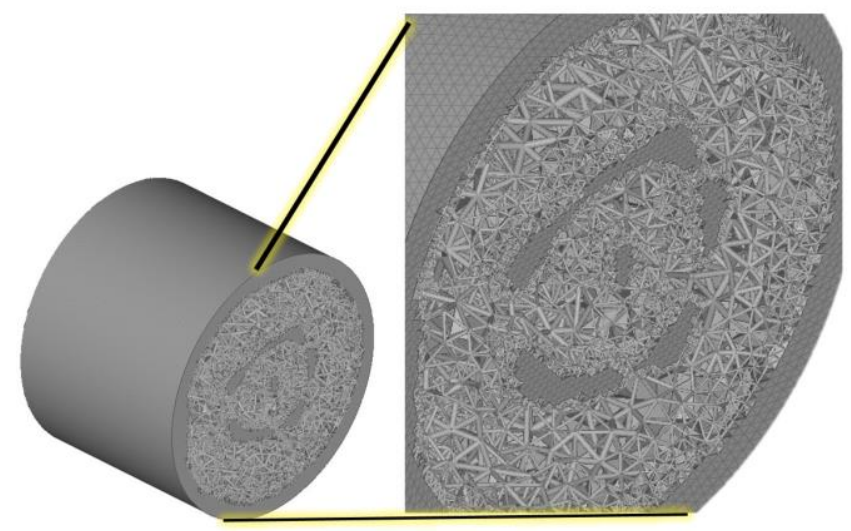

Fig. 4. CLS design for support stiffness maximization.

Shape optimization can address the designed complex objectives and boundary conditions. Rapid prototyping is a promoting technology in civil engineering ${ }^{(28,29)}$. The use of rapid prototyping is giving the opportunity to new design possibilities. This work is examining the use of will known image processing method as a shape optimization updating methodology for minimal computational cost, and real applied applications.

\section{Numerical implementation}

In case of static loading, the support structure is suspending the spherical tank at the desired level. The design domain to be optimized is chosen to be the area below the tank, started from the lower half of it. A block is initially chosen to support the structure then, according to the objective function criteria, materials will be removed to give final feasible design, by topology optimization. In this work, compliance minimization, single stress, and p-norm function where addressed to optimized spherical support. Level set methods were used to optimize each case of objective function. The flow chart of working process is described in Figure 5. Optistruct solver is used to computing the finite element analysis results to submit it to the optimization process for the three objective function and methodologies.

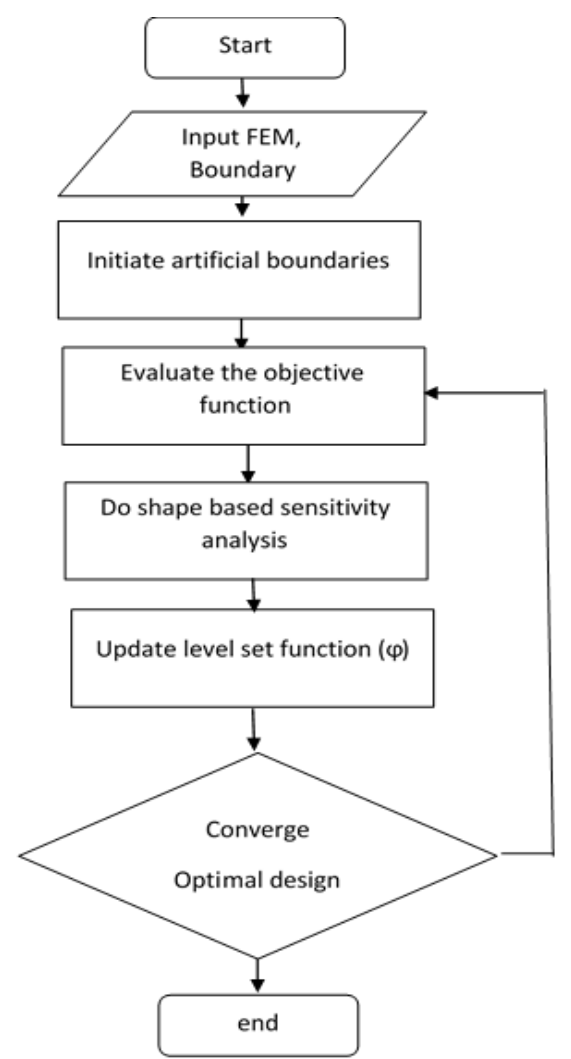

Fig. 5. Level set based shape optimization algorithm. 


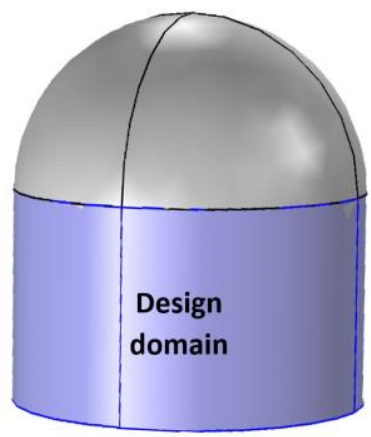

Fig. 6. Spherical Tank supports Initial design.

\section{Results}

Level set methods were used to optimize each case of objective function. Figure 7 shows the design of the support for maximum stiffness of the support. This is shows different design than the orthodox one (Figure.4). The objective function history showed smoothness and good objective achievements as shown in Figure 8. The final results of maximum stress minimization, and overall stress minimization (pnorm function ) are shown in Figure .9, and Figure .10. Figure 10 shows the final stress analyst
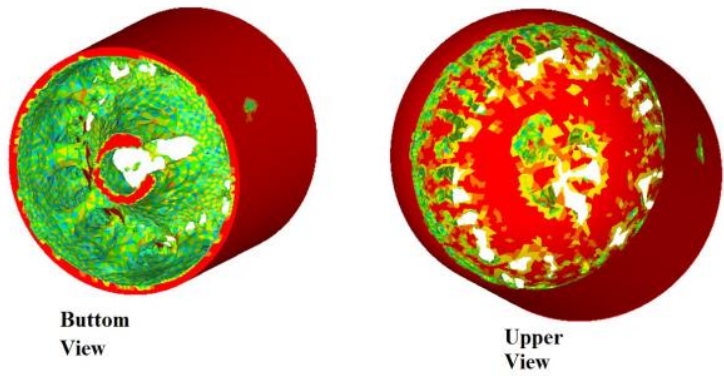

Fig. 7. Spherical Tank supports Initial design.

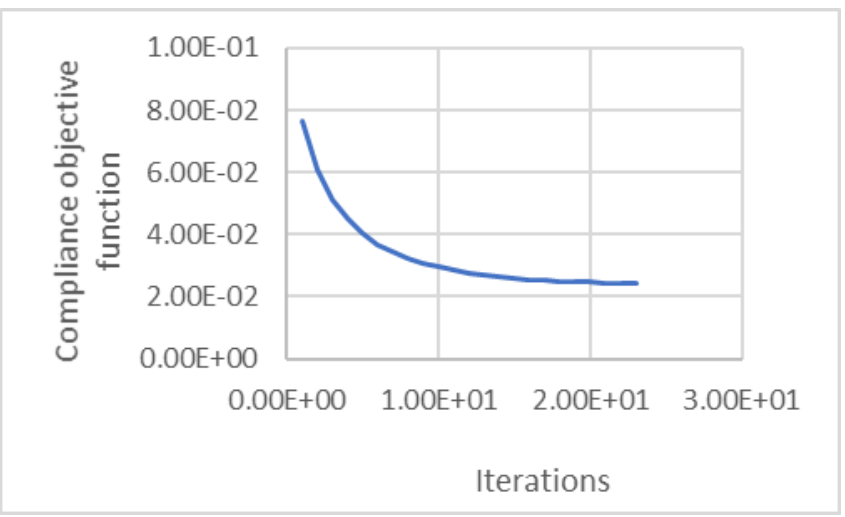

Fig. 8. Compliance objective function.

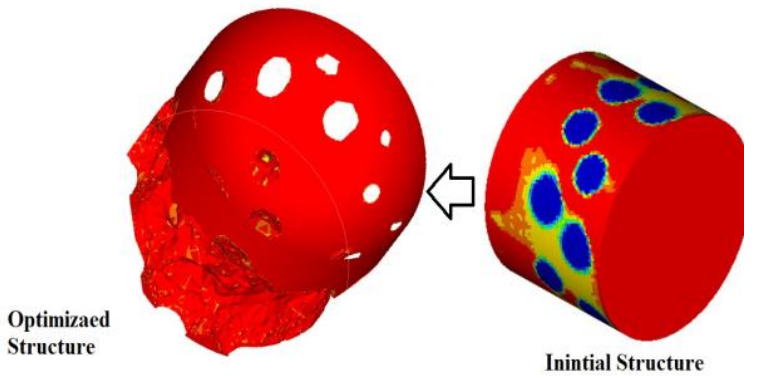

Fig. 9. Pnorm optimization results.

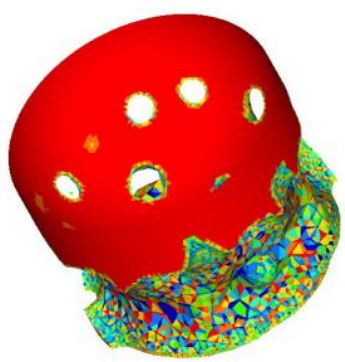

Fig. 10. Single stress optimization results.

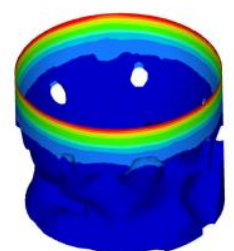

A

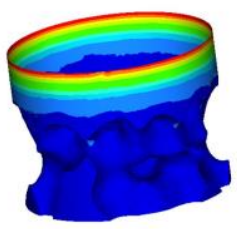

B

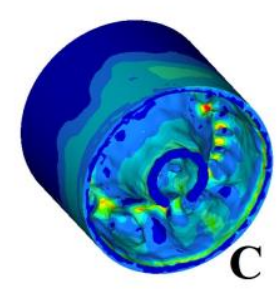

Fig. 11. Stress analyses for (A) Pnorm ;(B) Single stress (C) compliance optimization results.

Table.1 shows the maximum von Mises stress and the maximum displacement for the design structure. The design based on compliance shows particularly good results compared to the stress-based functions. This is not surprising such that the layout of the problem is hemispherical in shape. Therefore, compliance function tries to connect the loading elements to the supporting element following this hemispherical shape. This will limit the stress concentration areas.

Table 1. von Mises stress results of optimization cases.

\begin{tabular}{|l|l|}
\hline Structure & von Mises $\left(\mathrm{N} / \mathrm{mm}^{2}\right)$ \\
\hline Compliance & 16.59 \\
\hline Local Stress & 19.65 \\
\hline pnorm & 19.65 \\
\hline
\end{tabular}




\section{Conclusion}

In the case of spherical or rounded construction, Compliance objective function shows quite good results in increasing the design stiffness, without exhausting the structure with spots of stress concentration. The level set method gave good shapes, as a shape optimization method. The successfulness of Implementing Level set method with commercial software such as OptiStruc solver is promoting the gaining of new designs. This will improve the quality of non-parametric structural design and increase the feasibility of decreasing the cost.

\section{References}

(1) H. A. Eschenauer, C. Mattheck, and N. Olhoff, Engineering Optimization in Design Processes: Proceedings of the International Conference, Karlsruhe Nuclear Research Center, Germany, September 3-4, 1990 vol. 63: Springer Science \& Business Media, 2012.

(2) R. Fletcher, Practical methods of optimization: John Wiley \& Sons, 2013.

(3) A. Cherkaev, Variational methods for structural optimization vol. 140: Springer Science \& Business Media, 2012.

(4) K. Park and C. A. Felippa, "A variational principle for the formulation of partitioned structural systems," International Journal for Numerical Methods in Engineering, vol. 47, pp. 395-418, 2000.

(5) A. Abe, M. Nakamura, I. Sato, H. Uetani, and T. Fujitani, "Studies of the large-scale sea transportation of liquid hydrogen," International Journal of Hydrogen Energy, vol. 23, pp. 115-121, 1998.

(6) S. Mokhatab, J. Y. Mak, J. V. Valappil, and D. A. Wood, Handbook of liquefied natural gas: Gulf Professional Publishing, 2013.

(7) D. R. Moss, Pressure vessel design manual: Elsevier, 2004.

(8) M. Y. Wang, X. Wang, and D. Guo, "A level set method for structural topology optimization," Computer methods in applied mechanics and engineering, vol. 192, pp. 227-246, 2003.

(9) G. Allaire, F. Jouve, and A.-M. Toader, "Structural optimization using sensitivity analysis and a level-set method," Journal of computational physics, vol. 194, pp. 363-393, 2004.

(10) C. Groth, A. Chiappa, and M. Biancolini, "Shape optimization using structural adjoint and RBF mesh morphing," Procedia Structural Integrity, vol. 8, pp. 379-389, 2018.

(11) J. Yvonnet, H. L. Quang, and Q.-C. He, "An $\mathrm{XFEM} /$ level set approach to modelling surface/interface effects and to computing the size-dependent effective properties of nanocomposites," Computational Mechanics, vol. 42, pp. 119-131, 2008.

(12) J. Chessa, P. Smolinski, and T. Belytschko, "The extended finite element method (XFEM) for solidification problems," International Journal for Numerical Methods in Engineering, vol. 53, pp. 1959-1977, 2002.

(13) M. Farsad, F. J. Vernerey, and H. S. Park, "An extended finite element/level set method to study surface effects on the mechanical behavior and properties of nanomaterials," International Journal for Numerical Methods in Engineering, vol. 84, pp. 1466-1489, 2010.

(14) Q. Duan, J. H. Song, T. Menouillard, and T. Belytschko, "Element - local level set method for three - dimensional dynamic crack growth," International Journal for Numerical Methods in Engineering, vol. 80, pp. 1520-1543, 2009.

(15) G. Zi and T. Belytschko, "New crack - tip elements for XFEM and applications to cohesive cracks," International Journal for Numerical Methods in Engineering, vol. 57, pp. 2221-2240, 2003.

(16) M. Otomori, T. Yamada, K. Izui, and S. Nishiwaki, "Matlab code for a level set-based topology optimization method using a reaction diffusion equation," Structural and Multidisciplinary Optimization, vol. 51, pp. 1159-1172, 2015.

(17) S. Osher and J. A. Sethian, "Fronts propagating with curvature-dependent speed: algorithms based on Hamilton-Jacobi formulations," Journal of computational physics, vol. 79, pp. 12-49, 1988.

(18) F. Gibou, R. Fedkiw, and S. Osher, "A review of level-set methods and some recent applications," Journal of Computational Physics, 2017.

M. P. Bendsoe and O. Sigmund, Topology optimization: theory, methods, and applications: Springer Science \& Business Media, 2013. 
(20) P. Duysinx and M. P. Bendsøe, "Topology optimization of continuum structures with local stress constraints," International journal for numerical methods in engineering, vol. 43, pp. 1453-1478, 1998.

(21) C. Le, J. Norato, T. Bruns, C. Ha, and D. Tortorelli, "Stress-based topology optimization for continua," Structural and Multidisciplinary Optimization, vol. 41, pp. 605-620, 2010.

(22) E. Holmberg, B. Torstenfelt, and A. Klarbring, "Stress constrained topology optimization," Structural and Multidisciplinary Optimization, vol. 48, pp. 33-47, 2013.

(23) W. P. Ziemer, Weakly differentiable functions: Sobolev spaces and functions of bounded variation vol. 120: Springer Science \& Business Media, 2012.

(24) A. Verbart, "Topology Optimization with Stress Constraints," TU Delft, Delft University of Technology, 2015.

(25) V. Dakshnamoorthy, "Automated Lattice Optimization of Hinge Fitting with Displacement Constraint," 2016.

(26) N. S. Sripada, "A Methodology for Topology and Lattice Structure Optimization of a Cargo Drone Motor Mount," 2018.

(27) S. Daynes, S. Feih, W. F. Lu, and J. Wei, "sandwich structures with 3D printed Functionally graded lattice cores."

(28) M. Hambach and D. Volkmer, "Properties of 3D-printed fiber-reinforced Portland cement paste," Cement and Concrete Composites, vol. 79, pp. 62-70, 2017.

(29) S. Z. Jones, R. Buswell, W. L. de Silva, and J. Dirrenberger, "3D printing using concrete extrusion: a roadmap for research," 2018. 